\title{
Impacto da prática regular de exercício físico no equilíbrio, mobilidade funcional e risco de queda em idosos institucionalizados
}

\author{
Fernando Ribeiro ${ }^{1,2}$ \\ Sofia Gomes \\ Fantina Teixeira \\ Gabriela Brochado ${ }^{1,2}$ \\ José Oliveira ${ }^{1}$
}

https://doi.org/10.5628/rpcd.09.01.36

\author{
${ }^{1}$ Centro de Investigação em Actividade Física, Saúde e \\ Lazer (CIAFEL) \\ Faculdade de Desporto \\ Universidade do Porto \\ Portugal \\ ${ }^{2}$ Departamento de Fisioterapia \\ Escola Superior de Saúde do Vale do Sousa \\ Gandra \\ Portugal
}

\section{RESUMO}

O objectivo deste estudo foi comparar o equilíbrio, a mobilidade funcional e a proporção de sujeitos em categorias de risco de queda em função do desempenho nos testes de aptidão funcional, entre idosos treinados e não treinados. Adicionalmente, procurou-se determinar o risco de queda (odds ratio) em função de os idosos praticarem ou não exercício. 144 idosos institucionalizados foram divididos em dois grupos: o grupo de exercício $(n=65,76.7 \pm 8.1$ anos de idade) composto pelos idosos que reportaram praticar exercício físico estruturado supervisionado nos 12 meses anteriores ao início do estudo (sessões com 60 minutos de duração, pelo menos 3 vezes por semana); o grupo que não praticou exercício ( $\mathrm{n}=79,78.9 \pm 8.4$ anos de idade). As sessões eram compostas por exercícios dirigidos para o desenvolvimento da aptidão cardiorespiratória, do equilíbrio, da coordenação, da força muscular e da flexibilidade. Para avaliação do equilíbrio recorreu-se ao Functional Reach Test (FRT) e para a mobilidade funcional ao Timed Up \& Go Test (TUG). Idosos treinados (i) apresentam melhor performance no teste de equilíbrio $(19.3 \pm 9.6$ vs. $14.6 \pm 5.3 \mathrm{~cm} ; p<0.001)$ e mobilidade funcional ( $13.0 \pm 4.2$ vs. $17.6 \pm 7.5$ s; $p<0.001$ ), (ii) têm menor probabilidade de estarem em risco elevado de queda, expresso pelos valores do FRT (odds ratio, 0.21 , 95\% IC 0.068$0.629, \mathrm{p}=0.006$ ), e (iii) de apresentarem risco de queda nos próximos 5 anos, expresso pelos valores do TUG (odds ratio, $0.27,95 \%$ IC $0.126-0.582, \mathrm{p}=0.001)$. Em conclusão, idosos treinados apresentam melhor mobilidade funcional e equilíbrio com consequente diminuição do risco de queda do que idosos não treinados.

Palavras-Chave: idosos, equilíbrio, mobilidade funcional, exercício, quedas

\section{ABSTRACT \\ Impact of regular physical exercise participation in balance, func- tional mobility and fall risk in institutionalized older adults}

The purpose of this study was to compare balance, functional mobility and the proportion of subjects in each category of fall risk based on the values of the functional tests, between trained and untrained older adults. Additionally, one aimed to determine the odds ratio of fall risk for those who did not exercise comparatively to those who did. 144 institutionalized older adults were divided into two groups: the exercise group ( $n=65,76.7 \pm 8.1$ years of age) composed by older adults who reported the participation in regular physical exercise during the 12 months previous to the beginning of the study (60-minute sessions, at least 3 times per week); the group that did not participate in physical exercise $(n=79,78.9 \pm 8.4$ years of age). The exercise sessions were composed by aerobic, balance, coordination, flexibility and muscle strength exercises. Balance and functional mobility were assessed using the Functional Reach Test (FRT) and Timed Up \& Go Test (TUG), respectively. Trained older adults (i) performed better in the balance $(19.3 \pm 9.6$ vs. $14.6 \pm 5.3 \mathrm{~cm} ; p<0.001)$ and functional mobility tests $(13.0 \pm 4.2$ vs. $17.6 \pm 7.5 s ; p<0.001)$; (ii) were less likely to be classified as in high risk of fall, based on the results of FRT (OR, 0.21, 95\% CI 0.068-0.629, $p=0.006$ ), and (iii) were less likely to be classified as in risk of falling in the following 5 years, based on the results of TUG (OR, 0.27, 95\% CI 0.126-0.582, $p=0.001)$. In conclusion, trained older adults showed better balance and functional mobility, and, consequently, lower fall risk than untrained older adults.

Key-words: Elderly, balance, functional mobility, exercise, falls 


\section{INTRODUÇÃO}

O envelhecimento é acompanhado pelo marcado declínio das capacidades funcionais. Sendo um processo complexo, o envelhecimento resulta da interacção de diversos factores (p.e. factores genéticos, estilo de vida, doenças crónicas) determinando o declínio da capacidade funcional. No entanto, a manutenção de um estilo de vida activo através da realização regular de exercício físico (p.e. exercício aeróbio e/ou exercício de força) contribui para um envelhecimento mais saudável, que se caracteriza entre outros aspectos por níveis de aptidão que se relacionam com menor risco de morbilidade e mortalidade ${ }^{(1)}$.

O declínio da capacidade funcional resulta, em parte, de alterações neuromusculares tais como a desnervação muscular, a atrofia e perda selectiva de fibras musculares (especialmente das fibras tipo II) com redução da massa muscular total e a diminuição_da força e da potência muscular. Estas alterações repercutem-se negativamente no equilíbrio e na mobilidade funcional dos sujeitos idosos pela redução da eficácia dos mecanismos de ajustamento postural e do controlo motor (2), contribuindo para o aumento do risco de quedas e fracturas na população idosa(3). Risco esse que é particularmente elevado em idosos institucionalizados, uma vez que, entre outros factores, os níveis de aptidão funcional são inferiores aos de idosos não institucionalizados o que poderá explicar parcialmente a maior prevalência de quedas com fractura do colo do fémur que se tem observado neste segmento populacional $(4,5)$.

O padrão de declínio da força muscular não é uniforme em todas as regióes corporais e grupos musculares. A força dos membros inferiores diminui mais rapidamente com a idade do que a dos membros superiores. À diminuição da força dos membros inferiores associa-se menor capacidade de realização de certas acções e actividades da vida diária, tais como: levantar de uma cadeira, apanhar um objecto do chão, caminhar e subir escadas ${ }^{(1)}$. Contudo, vários autores sugerem que independentemente do sexo, a participação de idosos em programas de exercício físico promove aumento da massa muscular, da força muscular(1, 2, 6) e do equilíbrio(1), reduzindo o risco de quedas ${ }^{(1,7,8)}$ e consequentemente de fracturas ${ }^{(1)}$.
O aumento da população idosa e os elevados níveis de inactividade física que em diversos países se registam nesta população, níveis esses que são mais acentuados nos idosos institucionalizados(9), fazem prever o aumento da prevalência e incidência de quedas nesta faixa populacional. Deste modo, o exercício físico poderá desempenhar um papel chave na manutenção do equilíbrio, da mobilidade funcional e consequentemente na prevenção de quedas em idosos. Tendo em consideração o anteriormente exposto constituiu-se como objectivo deste estudo a comparação do equilíbrio, da mobilidade funcional e da proporção de sujeitos em categorias de risco de queda em função do desempenho nos testes de aptidão funcional, entre idosos institucionalizados treinados e não treinados. Adicionalmente, procurou-se determinar o risco de queda (odds ratio) em função de os idosos serem ou não praticantes de exercício.

\section{MATERIAL E MÉTODOS}

\section{Recrutamento e selecção dos participantes}

A amostra foi recrutada em Lares de $3^{\mathrm{a}}$ Idade, da área metropolitana da cidade do Porto, que ofereciam aos seus residentes programas de exercício físico. Os responsáveis dos Lares de $3^{\mathrm{a}}$ Idade foram contactados telefonicamente e convidados a aderirem ao estudo. Posteriormente, após obtida adesão dos responsáveis dos lares, foi efectuado convite aos residentes para participarem no estudo. Todos os que concordaram em participar no estudo foram entrevistados para recolher a história médica e determinar a sua elegibilidade para participar no estudo. Para ser incluído no estudo os sujeitos deveriam ser institucionalizados, com idade igual ou superior a 65 anos e não ter nenhum dos seguintes critérios de exclusão: lesões osteo-articulares ou músculo-tendinosas recentes envolvendo os membros inferiores; patologia vestibular e/ou do sistema nervoso central; hipotensão postural; patologia impeditiva da prática de exercício físico; e, estar a tomar medicação com influência no equilíbrio. Adicionalmente, a todos os voluntários foi aplicado um questionário para a determinação daqueles que praticavam regularmente exercício físico estruturado nos 12 meses anteriores ao inicio do estudo (sessões de 60 minutos pelo menos 3 vezes por semana) e daqueles que não pra- 
ticavam. As informações referentes à organização e estrutura das sessões de exercício foram obtidas junto dos responsáveis pela sua supervisão, recolhendo-se informação respeitante ao tipo de exercício, frequência semanal e duração das sessões. De acordo com as informações prestadas, as sessões dividiam-se em 3 fases, uma fase inicial de aquecimento, uma fase principal e uma fase final de retorno à calma. A parte principal das sessões era fundamentalmente composta por exercícios dirigidos para o desenvolvimento da aptidão cardiorespiratória, do equilíbrio, da coordenação, da força muscular e da flexibilidade.

\section{Amostra}

A amostra final foi constituída por 144 idosos institucionalizados, com idade compreendida entre $65 \mathrm{e}$ 96 anos. Os sujeitos foram divididos em dois grupos: exercício ( $n=65,51$ sujeitos do sexo feminino; idade: $76.7 \pm 8.1$ anos; peso: $73.9 \pm 15.2 \mathrm{~kg}$;_altura: 159.6 $\pm 7.8 \mathrm{~cm}$ ) e sem exercício $(n=79,51$ sujeitos do sexo feminino, idade:78.9 \pm 8.4 anos; peso: $70.1 \pm$ $12.8 \mathrm{~kg}$; altura: $158.2 \pm 8.5 \mathrm{~cm}$ ).

Todos os sujeitos deram o seu consentimento informado por escrito e todos os procedimentos foram efectuados de acordo com a declaração de Helsínquia.

\section{Avaliação do equilíbrio e da mobilidade funcional}

Todos os sujeitos completaram a recolha de dados numa única sessão matinal, após familiarização com o protocolo experimental e com os instrumentos de avaliação.

A avaliação do equilíbrio foi efectuada com recurso ao Functional Reach Test (FRT) e a mobilidade funcional ao Timed Up \& Go Test (TUG). Durante a realização dos testes todos os sujeitos foram instruídos a usar o mesmo tipo de calçado. Antes da avaliação, o examinador familiarizava o sujeito com os procedimentos através da explicação, demonstração e da realização de uma ou mais repetições práticas. Para cada teste, cada sujeito executou três repetições retirando-se a melhor das três para análise.

O FRT é um teste de performance que mede a distância máxima que um sujeito, na posição de pé, pode alcançar anteriormente mantendo os pés fixos.
Na posição inicial, o sujeito encontra-se de pé com o membro superior dominante numa posição de flexão do ombro a $90^{\circ}$, cotovelo em extensão e segurando um pequeno objecto cilíndrico na mão. Em seguida, é-lhe pedido que, com base de sustentação fixa, faça flexão do tronco projectando anteriormente o membro superior sem que os calcanhares deixem de estar em contacto com o solo. $\mathrm{O}$ valor do teste é definido pela distância percorrida pela mão ao deslocar-se horizontalmente à altura dos ombros. A distância foi medida numa fita métrica fixada na parede e colocada ao nível do acrómio. O ponto de referência para a medição inicial e final foi a extremidade distal do terceiro metacarpo ${ }^{(10)}$.

Mobilidade funcional é um termo usado para descrever o equilíbrio e a marcha usadas nas actividades da vida diária (p.e. sentar e levantar de uma cadeira, marcha, mudança de direcção). Para avaliar a mobilidade funcional com recurso ao TUG, foi dada instrução oral aos sujeitos para se levantarem de uma cadeira, caminhar três metros de forma segura e o mais rapidamente possível, atravessar uma linha marcada no chão, dar a volta, fazer o caminho inverso e voltar a sentar-se. Mede-se em segundos(11).

\section{Análise Estatística}

A análise estatística foi realizada utilizando o programa estatístico SPSS versão 16.0 (SPSS Inc., Chicago, IL, USA). Para a descrição dos dados foram usados os valores da média e desvio padrão. O teste de Kolmogorov-Smirnov foi utilizado para testar a normalidade de distribuição. Para comparação de médias entre grupos foi utilizado o teste $t$ independente. Numa segunda fase foram computadas: (i) uma variável binária com os valores de TUG, considerando os valores acima e abaixo de 16 segundos, e (ii) uma variável de FRT com 4 categorias, considerando os valores acima de $24.4 \mathrm{~cm}$, entre 15.24 e $25.40 \mathrm{~cm}$, abaixo de $15.24 \mathrm{~cm}$ e incapacidade de realizar o teste $(0 \mathrm{~cm})$. O valor de referência do TUG foi determinado tendo em consideração que um valor superior a 16 segundos é preditor de elevado risco de quedas num período de 5 anos (12). Relativamente aos valores de FRT, foi demonstrado que a capacidade de alcance superior a $24.4 \mathrm{~cm}$, entre $15.24-25.40 \mathrm{~cm}$ e inferior a $15.24 \mathrm{~cm}$, assim como a incapacidade de realizar o teste, correspondem a baixo, moderado, 
elevado e muito elevado risco de quedas $(10)$. O Teste do Chi-quadrado foi utilizado para analisar a proporção de idosos em cada categoria do FRT e do TUG, e a associação entre a prática de exercício e os valores de desempenho no FRT e no TUG. A probabilidade de ocorrência de quedas (em função da distribuição dos idosos pelas categorias acima descritas) associada à prática ou ausência de prática de exercício físico foi analisada através da regressão logística binária e da regressão logística multinomial. O nível de significância foi estabelecido em 5\%.

\section{RESULTADOS}

Os grupos não apresentam diferenças relativamente à idade, peso e altura. Idosos institucionalizados a realizar exercício físico estruturado nos 12 meses anteriores à realização do estudo apresentaram melhor equilíbrio, expresso nos valores do FRT $(19.3 \pm 9.6 \mathrm{~cm}$ vs. $14.6 \pm 5.3 \mathrm{~cm} ; p<0.001)$ (Figura 1 ), e melhor mobilidade funcional, expressa nos valores do TUG $(13.0 \pm 4.2 \mathrm{~s}$ vs. $17.6 \pm 7.5 \mathrm{~s} ; p$ $<0.001$ ) (Figura 2), do que idosos que não realizaram exercício físico estruturado durante o mesmo período de tempo. A leitura dos valores médios obtidos no FRT e no TUG permitem verificar que, em média, os idosos que realizaram exercício físico obtiveram $25 \%$ melhor performance no FRT e $35 \%$ melhor performance no TUG do que idosos que não participaram em programas de exercício.
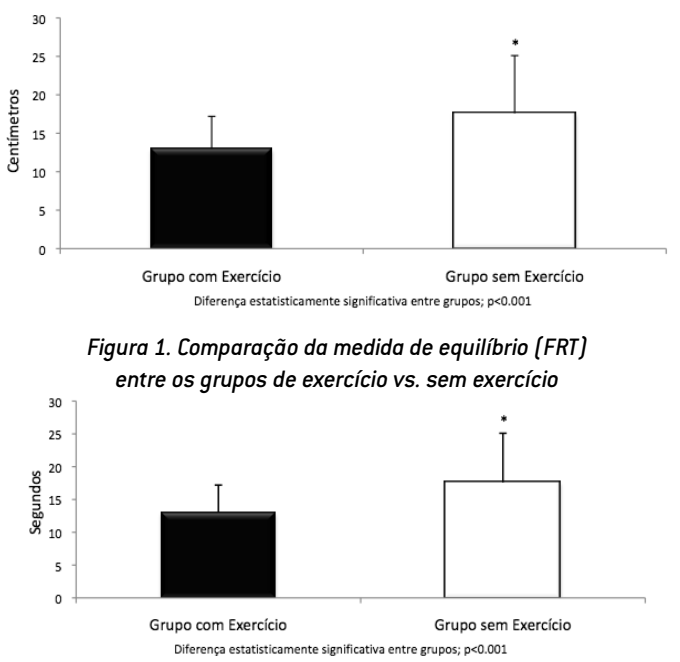

Figura 2. Comparação da medida de mobilidade funcional [TUG] entre os grupos de exercício vs. sem exercício
O número de idosos que apresenta risco de queda nos próximos 5 anos em função do desempenho no TUG é significativamente superior no grupo que não pratica exercício comparativamente ao grupo de exercício ( 12 vs. $36 ; \chi^{2}=12.0, \mathrm{df}=1, \mathrm{p}=0.001$ ). A comparação dos grupos por categorias de risco, em função do desempenho no FRT, revelou que o grupo de exercício apresenta um maior número de sujeitos em baixo risco de queda ( 14 vs. $5 ; \chi^{2}=4.26, \mathrm{df}=1$, $\mathrm{p}=0.039)$ e um menor número de sujeitos em elevado risco de queda (30 vs. $52 ; \chi^{2}=5.9, \mathrm{df}=1$, $\mathrm{p}=0.015$ ) (Tabela 1$)$. A prática de exercício apresenta uma associação significativa com o equilíbrio (FRT, $\chi^{2}=8,91 ; \mathrm{df}=2 ; \mathrm{p}=0.012 ; \phi^{2}=0.06$ representado um efeito médio) e com a mobilidade funcional (TUG, $\chi^{2}=11.8 ; \mathrm{df}=1 ; \mathrm{p}=0.001 ; \phi^{2}=0.07$ representado um efeito médio).

Os idosos que praticam exercício apresentam uma diminuição significativa do risco queda nos próximos 5 anos comparativamente aos idosos sem exercício (odds ratio, 0.27, 95\% IC 0.126-0.582, $\mathrm{p}=0.001$ ). A prática de exercício diminui a probabilidade de estar em risco de queda nos próximos 5 anos em $63 \%$. Da mesma forma, a prática regular de exercício diminui em $79 \%$ a probabilidade de apresentar risco elevado de queda, em função do desempenho no FRT (odds ratio, $0.21,95 \%$ IC 0.068-0.629, $\mathrm{p}=0.006$ ).

\section{DISCUSSÃO}

Os resultados deste estudo revelaram que idosos institucionalizados a participar regularmente em sessões de exercício físico supervisionado apresentam melhor equilíbrio e mobilidade funcional do que idosos não treinados, apresentando consequentemente menor risco de queda.

Os problemas de mobilidade em idosos estão geralmente associados a uma combinação de défices de equilíbrio, marcha e força muscular dos membros inferiores, défices esses que são também factor de risco de queda e de perda de autonomia para a realização das actividades da vida diária(13-15). De facto, as alterações associadas ao envelhecimento tais como o declínio da massa muscular e a diminuição da acuidade do sistema sensoriomotor são factores importantes associados à diminuição do equilíbrio e da mobilidade funcional que se observa em idosos ${ }^{16-}$ 18). Especialmente em idosos institucionalizados, que 
Tabela 1. Frequência absoluta e relativa [\%] de sujeitos dos dois grupos para cada categoria do risco de queda em função dos valores de desempenho no FRT e TUG

\begin{tabular}{lccccc}
\hline & Baixo & Risco de quedas (FRT) & Moderado & Elevado & \multicolumn{2}{c}{ Risco de queda a 5 anos (TUG) } \\
Sem risco & Em risco \\
\hline Grupo de exercício & $14(21.5)$ & $21(32.3)$ & $30(46.2)$ & $53(81.5)$ & $12(18.5)$ \\
\hline Grupo sem exercício & $5(6.3)^{*}$ & $22(27.8)$ & $52(65.8) \dagger$ & $43(54.4)$ & $36(45.6) \#$ \\
\hline
\end{tabular}

* diferença estatisticamente significativa para o grupo de exercício, $p=0.039$; $†$ diferença estatisticamente significativa para o grupo de exercício, $p=0.015$; \# diferença estatisticamente significativa para o grupo de exercício, $p=0.001$.

por estarem menos envolvidos nas rotinas das actividades diárias ou porque à partida apresentam patologias crónico-degenerativas que condicionam uma vida autónoma e independente, a força muscular pode-se deteriorar até um ponto em que se torna difícil ser independente na marcha e nas actividades da vida diária(19). No entanto, a participação regular em programas de exercício revela-se uma intervenção efectiva na redução/prevenção do declínio funcional associado ao envelhecimento(1), existindo evidência de que programas de exercício físico que incluam o fortalecimento muscular dos membros inferiores e o treino de equilíbrio melhoram a função física, o equilíbrio, a mobilidade funcional (p.e. velocidade da marcha, transferências, subir escadas e levantar-se a partir da posição de sentado) e reduzem o risco de quedas $(7,20-23)$.

È expectável que melhor equilíbrio e mobilidade funcional se repercuta no risco de queda. De facto, a interpretação em categorias dos valores de equilíbrio e mobilidade funcional, segundo os pontos de corte propostos Duncan et al.(10) e Okumiya et al.(12) respectivamente, suportar esta concepção. Os idosos do grupo de exercício apresentaram menor probabilidade de serem classificados como apresentando risco elevado de queda e de estarem em risco de sofrer uma queda nos próximos 5 anos do que os idosos do grupo sem exercício. Estes dados revestem-se de particular importância pelo facto das quedas serem um grave problema de saúde pública que se estima afectar uma em cada três pessoas com mais de 65 anos(24). A queda apresenta numerosas consequências directas e indirectas, que vão desde fracturas e contusões, até à incapacidade física a longo termo requerendo longos períodos de reabilitação, dependência e institucionalização. O risco aumentado de queda associa-se positivamente a várias alterações decorrentes do processo de envelhecimento, tais como diminuição da força muscular, flexibilidade, input sensorial, coordenação e equilíbrio $(25,26)$. Estima-se que $10 \%$ a $25 \%$ das quedas estejam associadas a défices de equilíbrio e anomalias no padrão de marcha(21). Contudo, é importante observar que estes factores de risco de queda são influenciados pela ausência de hábitos regulares de exercício físi$\mathrm{co}^{(27,28)}$. De facto, vários estudos têm demonstrado a eficácia de programas de exercício não só na redução do risco de queda (29-31), como também na redução do medo de cair $(32,33)$. Este aspecto também se reveste de extrema importância uma vez que o medo de cair pode levar à restrição da actividade, ao isolamento social, à diminuição da função física e da qualidade de vida, podendo o exercício físico desta forma fraccionar o ciclo vicioso negativo existente entre o medo de cair, a inactividade física e o risco aumentado de quedas(34).

No contexto da população idosa institucionalizada, a deterioração da aptidão funcional e o risco de queda são especialmente elevados $(4,5,19)$, pelo que os resultados deste estudo são particularmente encorajadores na exacta medida em que aqueles que realizam regularmente exercício físico têm menor risco de queda muito provavelmente pelo facto de serem funcionalmente mais aptos.

Uma das limitações do presente estudo advém do facto de não se ter avaliado a experiência anterior de queda e o medo de cair, tentando estabelecer uma associação entre os níveis de aptidão nos itens avaliados e a experiência de queda e o medo de cair nos idosos de ambos os grupos. Limitação esta que deverá ser tida em consideração na realização de futuros estudos. Da mesma forma, futuros estudos nesta 
área específica de conhecimento devem avaliar o efeito de diferentes programas de exercício, procurando estabelecer o tipo de exercício mais benéfico a curto e longo prazo.

Em conclusão, este estudo apresenta resultados complementares à evidência que suporta a efectividade de programas de exercício físico como estratégia terapêutica na prevenção de quedas, uma vez que demonstra que idosos institucionalizados a participar regularmente em programas estruturados de exercício físico, apresentam melhor mobilidade funcional, melhor equilíbrio e menor risco de queda do que idosos não treinados.

\section{CORRESPONDÊNCIA}

\section{Fernando Ribeiro}

Universidade do Porto

Faculdade de Desporto

Centro de Investigação em Actividade Física,

Saúde e Lazer

Rua Dr. Plácido Costa, 91

4200-450 Porto

Portugal

E-mail: fernando.silva.ribeiro@gmail.com 


\section{REFERÊNCIAS}

1. Mazzeo R, Cavanagh P, Evans W, Fiatarone M, Hagberg J, McAuley E , Startzell J (1998). American College of Sports Medicine Position Stand. Exercise and physical activity for older adults. Med Sci Sports Exerc 30(6): 992-1008

2. Timiras PS (2003). Physiological Basis of Aging and Geriatrics. $3^{\text {rd }}$ Edition. Boca Raton: CRC Press LLC.

3. Douris P, Southard V, Varga C, Schauss W, Gennaro C , Reiss A (2003). The Effect of Land and Aquatic Exercise on Balance Scores in Older Adults. J Geriatr Phys Ther 26(1): 3-6

4. Norton R, Campbell AJ, Reid IR, Butler M, Currie R, Robinson E , Gray H (1999). Residential status and risk of hip fracture. Age Ageing 28(2): 135-9

5. Ramnemark A, Nilsson M, Borssen B, Gustafson Y (2000). Stroke, a major and increasing risk factor for femoral neck fracture. Stroke 31(7): 1572-7

6. Krebs DE, Jette AM , Assmann SF (1998). Moderate exercise improves gait stability in disabled elders. Arch Phys Med Rehabil 79(12): 1489-95

7. Chandler JM, Duncan PW, Kochersberger G, Studenski S (1998). Is lower extremity strength gain associated with improvement in physical performance and disability in frail, community-dwelling elders? Arch Phys Med Rehabil 79(1): 24-30

8. Mecagni C, Smith JP, Roberts KE , O'Sullivan SB (2000). Balance and ankle range of motion in community-dwelling women aged 64 to 87 years: a correlational study. Phys Ther 80(10): 1004-11

9. Henry CJ, Webster-Gandy J , Varakamin C (2001). A comparison of physical activity levels in two contrasting elderly populations in Thailand. Am J Hum Biol 13(3): 310-5

10. Duncan PW, Weiner DK, Chandler J, Studenski S (1990). Functional reach: a new clinical measure of balance. $J$ Gerontol 45(6): M192-7

11. Podsiadlo D , Richardson S (1991). The timed "Up \& Go": a test of basic functional mobility for frail elderly persons. J Am Geriatr Soc 39(2): 142-8

12. Okumiya K, Matsubayashi K, Nakamura T, Fujisawa M, Osaki Y, Doi Y , Ozawa T (1998). The timed “up \& go" test is a useful predictor of falls in community-dwelling older people. J Am Geriatr Soc 46(7): 928-30

13. Guralnik JM, Ferrucci L, Simonsick EM, Salive ME , Wallace RB (1995). Lower-extremity function in persons over the age of 70 years as a predictor of subsequent disability. N Engl J Med 332(9): 556-61

14. Myers AH, Young Y, Langlois JA (1996). Prevention of falls in the elderly. Bone 18(1 Suppl): 87S-101S

15. Tinetti ME (2003). Clinical practice. Preventing falls in elderly persons. $N$ Engl J Med 348(1): 42-9

16. Lord SR, Ward JA (1994). Age-associated differences in sensori-motor function and balance in community dwelling women. Age Ageing 23(6): 452-60

17. Choy NL, Brauer S, Nitz J (2003). Changes in postural stability in women aged 20 to 80 years. J Gerontol A Biol Sci Med Sci 58(6): 525-30

18. Maki BE, Holliday PJ, Topper AK (1994). A prospective study of postural balance and risk of falling in an ambulatory and independent elderly population. J Gerontol 49(2): M72-84
19. Bautmans I, Van Hees E, Lemper JC, Mets T (2005). The feasibility of Whole Body Vibration in institutionalised elderly persons and its influence on muscle performance, balance and mobility: a randomised controlled trial [ISRCTN62535013]. BMC Geriatr 5: 17

20. Nelson ME, Fiatarone MA, Morganti CM, Trice I, Greenberg RA , Evans WJ (1994). Effects of high-intensity strength training on multiple risk factors for osteoporotic fractures. A randomized controlled trial. JAMA 272(24): 1909-14

21. Shumway-Cook A, Gruber W, Baldwin M, Liao S (1997). The effect of multidimensional exercises on balance, mobility, and fall risk in community-dwelling older adults. Phys Ther 77(1): 46-57

22. Liu-Ambrose T, Khan KM, Eng JJ, Janssen PA, Lord SR , McKay HA (2004). Resistance and agility training reduce fall risk in women aged 75 to 85 with low bone mass: a 6 month randomized, controlled trial. J Am Geriatr Soc 52(5): 657-65

23. Ribeiro F, Teixeira F, Brochado G, Oliveira J (2009). Impact of low cost strength training of dorsi- and plantar flexors on balance and functional mobility in institutionalized elderly people. Geriatr Gerontol Int 9: 75-80

24. Lewis SC (2003). Elder care in occupational therapy. $2^{\text {nd }}$ edition. Thorofare, NJ: Slack, Inc, 12-15, 251-258

25. Lord SR, Menz HB, Tiedemann A (2003). A physiological profile approach to falls risk assessment and prevention. Phys Ther 83(3): 237-52

26. Rubenstein LZ, Josephson KR (2002). The epidemiology of falls and syncope. Clin Geriatr Med 18(2): 141-58

27. Fleming BE, Pendergast DR (1993). Physical condition, activity pattern, and environment as factors in falls by adult care facility residents. Arch Phys Med Rehabil 74(6): 627-30

28. Kiely DK, Kiel DP, Burrows AB, Lipsitz LA (1998). Identifying nursing home residents at risk for falling. $J \mathrm{Am}$ Geriatr Soc 46(5): 551-5

29. DeVito CA, Morgan RO, Duque M, Abdel-Moty E, Virnig BA (2003). Physical performance effects of low-intensity exercise among clinically defined high-risk elders. Gerontology 49(3): 146-54

30. Becker C, Kron M, Lindemann U, Sturm E, Eichner B, Walter-Jung B , Nikolaus T (2003). Effectiveness of a multifaceted intervention on falls in nursing home residents. $J$ Am Geriatr Soc 51(3): 306-13

31. Day L, Fildes B, Gordon I, Fitzharris M, Flamer H, Lord S (2002). Randomised factorial trial of falls prevention among older people living in their own homes. BMJ 325(7356): 128

32. Verfaillie DF, Nichols JF, Turkel E, Hovell MF (1997). Effects of resistance, balance and gait training on reduction of risk factors leading to falls in elders. J Aging Phys Activity 5: 213-228

33. Carvalho J, Pinto J , Mota J (2007). Actividade física, equilíbrio e medo de cair. Um estudo em idosos institucionalizados. Rev Port Cien Desp 7(2): 225-231

34. Carter ND, Kannus P, Khan KM (2001). Exercise in the prevention of falls in older people: a systematic literature review examining the rationale and the evidence. Sports Med 31(6): 427-38 